\title{
Adolescents et jeunes adultes : vivre pendant et après un cancer
}

\section{Lemaitre $\cdot$ E. Seigneur}

(C) Springer-Verlag 2009
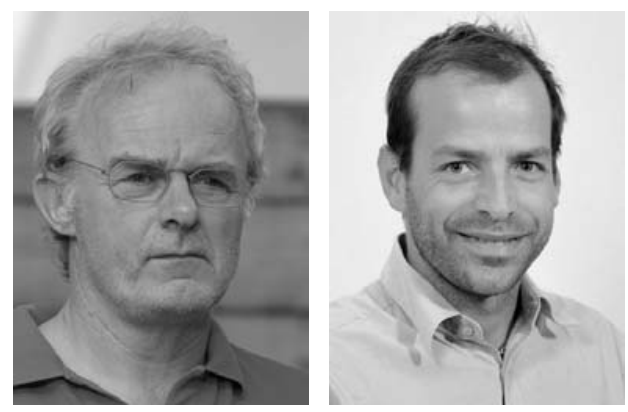

En France, chaque année, environ 1700 jeunes, âgés de 15 à 25 ans, apprennent qu'ils sont atteints d'un cancer. L'épidémiologie de ces cancers est très spécifique, et les taux de guérison sont plus faibles que pour les enfants dans certaines pathologies en raison probablement de certaines caractéristiques cliniques et biologiques de ces tumeurs, mais également du fait des disparités dans le parcours de soins des adolescents et jeunes adultes.

En effet, traités soit dans des services de pédiatrie ou des services d'adultes, ces jeunes patients ne trouvent pas toujours un environnement de soins adapté à leurs problématiques spécifiques. Accueillir et soigner des adolescents ou jeunes adultes gravement malades nécessitent un intérêt mais aussi des compétences et donc une formation à ces particularités. Celles-ci découlent principalement de la rencontre brutale entre la fragilité de cette période de transition que sont l'adolescence et le début de la vie adulte, et les enjeux de la survenue du cancer et de son traitement. L'ensemble des transformations physiques, psychiques et relationnelles propres à cette période de la vie va, en effet, être profondément et durablement remanié par le cancer et les menaces sur l'avenir qu'il fait peser, compliquant encore plus ce que l'on a coutume d'appeler le « travail » et la « crise » de l'adolescence. La construction identitaire, la dépendance aux autres, l'accès à l'autonomie affective et matérielle, les mouvements identificatoires constituent quelques-unes des problématiques compliquées par la survenue du cancer, en n'oubliant pas que ces jeunes patients ont aussi une histoire personnelle et familiale préalable à la maladie qui est source parfois de difficultés supplémentaires.

Cependant, il ne faudrait pas seulement réduire cette période de la vie à des problèmes ou à des conflits entre les

\footnotetext{
L. Lemaitre $(\bowtie)$

Service d'oncohématologie pédiatrique,

CHRU de Montpellier, 191, avenue du Doyen-Gaston-Giraud, F-34295 Montpellier, France

e-mail : 1-lemaitre@chu-montpellier.fr

E. Seigneur

Département d'oncologie pédiatrique, institut Curie,

26, rue d'Ulm, F-75248 Paris, France

etienne.seigneur@curie.net
}

jeunes eux-mêmes, leurs proches ainsi que les soignants qui s'en occupent. L'adolescence porte en elle également une richesse, richesse qui côtoie sa fragilité et qui est directement en rapport avec le caractère profondément dynamique de cette période. L'adolescence et le début de la vie adulte représentent la période des possibles, celle des acquisitions et des choix de vie pour l'avenir. Il est donc impératif également de soutenir et de permettre que ces potentialités puissent émerger en dépit de la maladie, ce qui implique pour les soignants un questionnement sur les conséquences à moyen et long terme des traitements délivrés.

L'évocation de cette dimension temporelle des soins ouvre une autre question d'actualité qui concerne le suivi et le devenir à long terme des patients guéris du cancer. Les progrès thérapeutiques en oncohématologie pédiatrique, majeurs ces 30 dernières années, ont permis une amélioration considérable des taux de guérison. Actuellement, environ trois enfants et adolescents sur quatre vont guérir de leur maladie, sachant que la guérison « médicale » du cancer n'exclut pas la présence de séquelles, physiques ou psychologiques, parfois invalidantes. On estime qu'environ une personne, âgée de 20 à 45 ans, sur 850 en France a survécu à un cancer survenu durant l'enfance ou l'adolescence, ce qui représente 25000 individus. Ainsi, l'organisation de la prévention, du dépistage et du suivi des séquelles représente donc un véritable enjeu de santé publique qui dépasse largement la seule question du soutien psychologique de ces personnes.

La Société française de psycho-oncologie (SFPO) a décidé cette année de consacrer son congrès annuel à toutes ces questions relatives aux adolescents et jeunes adultes atteints de cancer ou l'ayant été préalablement. Le comité scientifique a été élaboré dans une perspective multidisciplinaire, à l'image du dialogue nécessaire entre disciplines soignantes ou non auquel les adolescents nous obligent. Le programme scientifique a été conçu et sélectionné dans un souci de rencontre, de partage et de confrontation : entre psys et soignants non psys, entre professionnels de l'oncohématologie pédiatrique et adulte, entre professionnels intervenants auprès des adolescents et jeunes adultes malades et ceux assurant le suivi médical et psychologique à long terme et enfin entre soignants du monde hospitalier et ceux travaillant au domicile, en libéral ou encore au sein des réseaux de santé. Bien 
évidemment, les représentants des associations de patients, jeunes malades, parents et proches, apporteront un éclairage précieux et vivant à tous ces échanges.

Les ateliers de la SFPO se dérouleront en préambule à ce congrès. Ils permettront des échanges interactifs en petits groupes autour de thématiques cliniques qui nous sont apparues pertinentes au regard de la thématique du congrès.
Puissent ces journées et ces rencontres s'avérer amicales et fécondes afin de progresser dans notre compréhension et nos représentations de ce que vivent les adolescents et jeunes adultes confrontés au cancer. Nous souhaitons également qu'elles permettent de nourrir la réflexion autour des lieux et pratiques de soins pendant et après la maladie. Dans cette attente, nous vous y convions chaleureusement. 